\section{A4.9 HOW OSTEOBLAST REGULATES ENERGY METABOLISM AND SYSTEMIC INFLAMMATION DEPENDENT OF FRA-2 EXPRESSION}

doi:10.1136/annrheumdis-2013-203217.9

${ }^{1}$ Aline Bozec, 'Yubin Luo, 'Christine Zech, ${ }^{2}$ Maria Jimenez, 'Latifa Bakiri, 'Mirco Petrucelli, ${ }^{3}$ Michael Amling, ${ }^{1}$ Georg Schett, ${ }^{2}$ Erwin Wagner. ${ }^{1}$ Department of Medicine 3 , Rheumatology and Immunology, University of Erlangen-Nuremberg, Nikolaus-FiebigerZentrumGlueckstrasse 6, D-91054 Erlangen, Germany; ${ }^{2}$ Genes, Development and Disease Group, F-BBVA-CNIO Cancer Cell Biology Program, Spanish National Cancer Research Centre (CNIO), E-28029 Madrid; ${ }^{3}$ Department of Osteology and Biomechanics, University Medical Center, Hamburg-Eppendorf, Martinistrasse 52, D-20246 Hamburg, Germany

Background and Objectives The transcription factor Fra-2 (Fosl2) is a member of the AP-1 complex and an important regulator of bone homeostasis. We have previously shown that Fra-2 controls bone development, osteoclast size [1] and osteoblast differentiation through direct regulation of Collagen $1 \mathrm{a} 2$ and Osteocalcin (Ocn) [2] Recent studies have established that the skeleton functions as an endocrine organ affecting metabolism through Ocn [3], although only few transcription factors and only one osteoblast-derived hormone are known to affect the crosstalk between bone and metabolism.

Materials and Methods We have generated mice with specific deletion of Fra-2 (Fosl2) or ectopic expression of Fra-2 in osteoblast to study the role of Fra- 2 beyond the bone e.g. in metabolism.

Results Here we show that mice with osteoblast specific deletion of Fra-2 (Fosl2) have despite a low bone mass, an increased body weight. In contrast, ectopic expression of Fra-2 in osteoblasts display increased bone mass and decreased body weight accompanied with reduced serum glucose and insulin levels, improved glucose tolerance and insulin sensitivity. In addition, these Fra- 2 mutant mice are protected from metabolic impairment, when challenged with high fat diet (HFD). Surprisingly a systemic inflammation and macrophage infiltration in liver, spleen and lung was observed in Fra-2 osteoblast specific mice. Mechanistically, we showed that in osteoblasts Fra-2 transcriptionally represses an important adipocytokine Adiponectin (Adipoq), while it induces $O \mathrm{cn}$, both responsible for the glucose and insulin metabolism alteration. Whereas, the systemic inflammation was likely due to the transcriptional increased of Osteopontin (OPN) expression by Fra-2, which is known as a potent inductor of macrophage activation.

Conclusions Taking together these results show that Fra-2 expression in osteoblast transcriptionally modulates Adipoq, Ocn and OPN expression and secretion representing a novel mechanism for the endocrine function of the skeleton on systemic metabolism and inflammation.

\section{References}

1. Bozec A, et al, (2008). Osteoclast size is controlled by Fra-2 through LIF/LIF-receptor signalling and hypoxia. Nature 454(7201):221-225.

2. Bozec A, et al, (2010). Fra-2/AP-1 controls bone formation by regulating osteoblast differentiation and collagen production. The Journal of cell biology 190(6):1093-1106.

3. Karsenty G \& Ferron M (2012). The contribution of bone to wholeorganism physiology. Nature 481(7381):314-320.

\section{A4.10 HYDROGEN SULFIDE ATTENUATES STORE-OPERATED $\mathrm{Ca}^{2+}$ ENTRY IN ENDOTHELIAL AND SMOOTH MUSCLE CELLS}

doi:10.1136/annrheumdis-2013-203217.10

'B Kloesch, ${ }^{2} \mathrm{~S}$ Stranimaier, ${ }^{1,3} \mathrm{G}$ Steiner, ${ }^{2} \mathrm{~K}$ Schmidt. 'Ludwig Boltzmann Cluster Rheumatology, Balneology and Rehabilitation, Ludwig Boltzmann Institute of Rheumatology and Balneology, Kurbadstrasse 14, 1100 Vienna, Austria; ${ }^{2}$ Karl-FranzensUniversity Graz, Department of Pharmacology and Toxicology, Universitätsplatz 2, 8010 Graz, Austria; ${ }^{3}$ Medical University Vienna, Department of Internal Medicine III, Division of Rheumatology, Währinger Gürtel 18, 1090 Vienna, Austria
Background and Objectives Endothelial cells are active participants in inflammatory processes. They are involved in diverse activities including the regulation of leucocyte extravasation, angiogenesis, cytokine production, protease and extracellular matrix synthesis, vasodilation, etc. The small gaseous molecule hydrogen sulphide $\left(\mathrm{H}_{2} \mathrm{~S}\right)$ is involved in a variety of physiological processes like vascular relaxation, angiogenesis, neurotransmission and inflammation. In the vascular system, ATP-sensitive $\mathrm{K}^{+}$-channels are a major target for $\mathrm{H}_{2} \mathrm{~S}$ but over the last few years evidence has accumulated that several $\mathrm{Na}^{+}$- and $\mathrm{Ca}^{2+}$-permeable channels are also sensitive to $\mathrm{H}_{2} \mathrm{~S}$. In the present study we investigated the effect of $\mathrm{H}_{2} \mathrm{~S}$ on $\mathrm{Ca}^{2+}$ signalling in cultured endothelial and smooth muscle cells with special emphasis given to the role of $\mathrm{H}_{2} \mathrm{~S}$ in modulating store-operated $\mathrm{Ca}^{2+}$ channels.

Materials and Methods Experiments were performed with human microvascular endothelial cells (HMEC-1), endothelial cells isolated from porcine aorta, and smooth muscle cells isolated from rat aorta and rat trachea. Mobilisation of intracellular $\mathrm{Ca}^{2+}$ and $\mathrm{Ca}^{2+}$ entry was monitored by measuring the intracellular free $\mathrm{Ca}^{2+}$ concentration with FURA-2 in the absence and presence extracellular $\mathrm{Ca}^{2+}$, respectively. Activity of endothelial nitric oxide synthase (eNOS) in intact cells was determined as conversion of incorporated $\mathrm{L}$ - $\left[{ }^{3} \mathrm{H}\right]$-arginine into L- $\left[{ }^{3} \mathrm{H}\right]$-citrulline.

Results Incubation of human and porcine endothelial cells with the $\mathrm{H}_{2} \mathrm{~S}$-donor $\mathrm{NaHS}(100 \mu \mathrm{M}, 10-45 \mathrm{~min})$ evoked a release of $\mathrm{Ca}^{2+}$ from intracellular stores that was not accompanied by $\mathrm{Ca}^{2+}$ influx from the extracellular space. In accordance with these data suggesting that $\mathrm{H}_{2} \mathrm{~S}$ may inhibit store-operated $\mathrm{Ca}^{2+}$ entry, incubation of cells with $\mathrm{NaHS}$ attenuated $\mathrm{Ca}^{2+}$ influx induced by depletion of $\mathrm{Ca}^{2+}$ stores with receptor agonists (ATP, histamine) or the endoplasmatic reticulase ATPase inhibitor, thapsigargin. As a consequence, the stimulatory effect of these agonists on endothelial NO formation was strongly diminished, whereas the response to the $\mathrm{Ca}^{2+}$ ionophore A23187 was barely affected. Similar to the results obtained with endothelial cells, depletion of intracellular $\mathrm{Ca}^{2+}$ stores in smooth cells isolated from rat aorta or rat trachea also resulted in a pronounced $\mathrm{Ca}^{2+}$ entry that was completely blocked upon pretreatment of cells with $\mathrm{NaHS}$

Conclusions $\mathrm{H}_{2} \mathrm{~S}$ inhibits the stimulatory effect of $\mathrm{Ca}^{2+}$ of mobilising agonists on endothelial $\mathrm{NO}$ formation by attenuating storeoperated $\mathrm{Ca}^{2+}$ entry. Inhibition of store-operated $\mathrm{Ca}^{2+}$ channels by $\mathrm{H}_{2} \mathrm{~S}$ is not peculiarity of endothelial cells but also occurs in vascular and tracheal smooth muscle cells. These hitherto undescribed effects may be in part possible for the beneficial effects of $\mathrm{H}_{2} \mathrm{~S}$ in sulphur bath therapy.

\section{A4.11 BASELINE ELEVATED SERUM LEVELS OF CALPROTECTIN AS INDEPENDENT MARKER FOR RADIOGRAPHIC SPINAL PROGRESSION IN ANKYLOSING SPONDYLITIS}

doi:10.1136/annrheumdis-2013-203217.11

'Maureen Turina, ${ }^{2} J$ oachim Sieper, ${ }^{1,3}$ Nataliya Yeremenko, ${ }^{2}$ Hildrun Haibel, ${ }^{1,3}$ Dominique Baeten, ${ }^{2}$ Denis Poddubnyy. ${ }^{1}$ Department of Clinical Immunology and Rheumatology, Academic Medical Center/University of Amsterdam, The Netherlands; ${ }^{2}$ Department of Rheumatology, Charité, Campus Benjamin Franklin, Berlin, Germany; '3aboratory of Experimental Immunology, Academic Medical Center/University of Amsterdam, The Netherlands

Background and Objective Syndesmophytes formation and complete fusion of the total spine are common characteristics leading to functional impairment and disability in ankylosing spondylitis (AS) patients. Predictors for progression of structural damage are smoking, elevated levels of acute phase reactants and the presence of syndesmophytes at baseline. These predictors identify increased risk for progression at group level but their specificity is not strong enough to be used as biomarkers in individual patients. We recently 